CU-TP-598

\title{
Color Diffusion and Conductivity in a Quark-Gluon Plasma 8
}

\author{
Alexei V. Selikhov|尸 and Miklos Gyulassy \\ Department of Physics, Columbia University, New York, NY10027
}

June 1993

\begin{abstract}
Color diffusion is shown to be an important dissipative property of quark-gluon plasmas that rapidly damps collective color modes. We derive the characteristic color relaxation time scale, $t_{c} \approx\left(3 \alpha_{s} T \log \left(m_{E} / m_{M}\right)\right)^{-1}$, showing its sensitivity to the ratio of the static color electric and magnetic screening masses. This leads to a surprisingly small color conductivity, $\sigma_{c} \approx 2 T / \log \left(m_{E} / m_{M}\right)$, which in fact vanishes in the semiclassical (1-loop) limit.
\end{abstract}

The quark-gluon plasma (QGP) phase of QCD matter has been predicted to exhibit collective behavior similar to QED plasmas with Debye screened (color) electric interactions, collective plasmon oscillations, and collective color mode instabilities under a variety of non-equilibrium configurations (see, e.g., reviews in [1, 2, 3]). Such collective behavior of quark-gluon plasmas (QGP) may be observed experimentally via ultra-relativistic heavyion reactions if the time scales of dissipative processes that act to damp collectivity are sufficiently long. Thusfar, most studies of transport properties of QGP have focused on momentum relaxation processes to estimate viscosity and thermal conductivity coefficients, $d E / d x$, etc. [4, 5, [6]. Relaxation phenomena associated with the color degrees of freedom have received, on the other hand, less attention. In this letter, we derive a new transport coefficient, called color diffusion, that has no abelian analog. The related color relaxation time, $t_{c}$, measures how rapidly the color of a parton precesses in an ambient fluctuating background field.

\footnotetext{
1 This work was supported by the Director, Office of Energy Research, Division of Nuclear Physics of the Office of High Energy and Nuclear Physics of the U.S. Department of Energy under Contract No DE-FG-02-93ER-40764.

${ }^{2}$ Supported by the AUI/RHIC Fellowship Fund; Permanent address: Kurchatov Institute, 123182 Moscow D-182, Russia
} 
The relevance of $t_{c}$ can be seen most clearly in the context of color conductivity. In the relaxation time approximation, classical non-abelian transport theory leads to the following formula for the static color conductivity [7, 2]

$$
\sigma_{\mathrm{c}}=\omega_{p l}^{2} / \nu
$$

where $\omega_{\mathrm{pl}}^{2}=4 \pi \alpha_{\mathrm{s}} T^{2}\left(1+N_{f} / 6\right) / 3$ the color plasmon frequency [8, 9] and $\nu$ is a collision frequency. Color conductivity arises as a generalization of Ohm's law: $j_{i}^{a}=\sigma_{c} E_{i}^{a}$ and influences the evolution of a QGP through ohmic heating as pointed out in ref. [10]. Ohmic heating also couples mini-jets and beam jets in ultra-relativistic nuclear collisions as shown in ref.[11]. The main problem in this connection is to estimate the collision frequency, $\nu$. In QED plasmas, $\nu=1 / t_{p}$, where $t_{p}$ is the momentum relaxation time. In QCD, transport theory estimates for $t_{p}$ including color Debye and dynamical screening give [5]

$$
t_{p} \approx\left(4 \alpha_{\mathrm{s}}^{2} \ln \left(1 / \alpha_{\mathrm{s}}\right) T\right)^{-1}
$$

This time scale was used to estimate the magnitude of effects of ohmic heating in ref.[11]. We show below, however, that $t_{c}$ instead of $t_{p}$ controls the color conductivity, and that $t_{c} \ll t_{p}$ in the perturbative limit. In fact, we find that $t_{c}$ is closely related to the (divergent) gluon damping rate and that color diffusion strongly damps collective plasmon modes. We therefore find that non-abelian plasmas are surprisingly poor color conductors and that collective color modes are over-damped due to rapid color diffusion.

To derive $t_{c}$ we follow here ref. [12, 13] and consider the motion of a classical colored particle moving along a world line $x^{\mu}(\tau)$ with momentum $p^{\mu}(\tau)=m v^{\mu}(\tau)$ and $v^{\mu}=d x^{\mu} / d \tau$, that couples with an $N^{2}-1$ component color charge vector $\Lambda^{a}(\tau)$ to a background Yang-Mills field, $A_{\mu}^{a}(x)$. We follow this approach here because of its intuitive simplicity and because it leads to the same result that we find starting from the kinetic equations for the quark and gluon Wigner density matrices [1] including the Lenard-Balescu type collision terms derived in [14, 15]. While the derivation based on the later approach is more rigorous, it is also less transparent physically and therefore deferred to a subsequent paper 16.

Consider the motion of a heavy quark in the $d$ dimensional representation of $S U(N)$ with second order Casimir, $C_{2}$. The classical color charge vector is defined through the expectation value of the color current via

$$
j^{\mu a}(x)=g\left\langle\bar{\psi}(x) \gamma^{\mu} t^{a} \psi(x)\right\rangle=g \int d \tau v^{\mu}(\tau) \delta^{4}(x-x(\tau)) \Lambda^{a}(\tau),
$$

where $\left[t^{a}, t^{b}\right]=i f^{a b c} t^{c}$ and $t^{a} t^{a}=C_{2} \mathbf{1}$. Similarly, the classical momentum is related to the expectation value of the matter energy-momentum tensor

$$
T^{\mu \nu}(x)=i\left\langle\bar{\psi} \gamma^{\mu} D^{\nu}(x) \psi(x)\right\rangle=m \int d \tau v^{\mu}(\tau) v^{\nu}(\tau) \delta^{4}(x-x(\tau))
$$

where $D^{\nu}(x)=\partial^{\nu}+i g t^{a} A_{a}^{\nu}(x)$ The equations of motion follow from the conservation laws

$$
\partial_{\mu} T^{\mu \nu}=g j_{\mu}^{a} F_{a}^{\nu \mu}, \partial_{\mu} j^{\mu a}=g f^{a b c} A_{\mu}^{b} j^{\mu c} .
$$


Substituting Eqs. (3. (1) into the left and right hand side, we recover the equations derived in [12]:

$$
\begin{aligned}
\frac{d p^{\mu}}{d \tau} & =g v_{\nu} F_{a}^{\mu \nu} \Lambda^{a}, \\
\frac{d \Lambda^{a}}{d \tau} & =g f^{a b c} v^{\mu} A_{\mu}^{b} \Lambda^{c},
\end{aligned}
$$

where the fields are evaluated at $x^{\mu}(\tau)$. Since under a gauge transformation $U(x)=$ $\exp \left(i \theta^{a}(x) t^{a}\right), \psi \rightarrow U \psi$, the color charge must transform as $t^{a} \Lambda^{a}(\tau) \rightarrow U(x(\tau)) t^{a} \Lambda^{a}(\tau) U^{-1}(x(\tau))$, to insure the gauge covariance of eqs.(6.7). (Note that $m d / d \tau=p_{\mu} \partial^{\mu}$ along the world line.)

We study the time dependence of the color charge averaging over an ensemble of background field configurations. We assume that the ensemble characterizes a color neutral medium in which the $A_{\mu}^{a}$ are random fields such that the ensemble averaged potentials vanish in a suitable gauge. The ensemble average of Eq. (7) gives

$$
\frac{d}{d \tau}\left\langle\Lambda^{a}(\tau)\right\rangle=g f^{a b c}\left\langle[v A]_{\tau}^{b} \Lambda^{c}(\tau)\right\rangle,
$$

where for shorthand we write $[v A]_{\tau}^{b}=\left(p^{\mu}(\tau) / m\right) A_{\mu}^{b}(x(\tau))$. Integrating (formally) Eq. (7) and substituting the result back into Eq. (8), yields

$$
\frac{d}{d \tau}\left\langle\Lambda^{a}(\tau)\right\rangle=g^{2} f^{a b c} f^{c d e} \int_{0}^{\tau} d \tau^{\prime}\left\langle[p A]_{\tau}^{b}[p A]_{\tau^{\prime}}^{d} \Lambda^{e}\left(\tau^{\prime}\right)\right\rangle
$$

Our first main physical assumption is that the time scale of field fluctuations is short compared to the time scale of variations of the color orientation. Formally, this is suggested from eq.(9) if $g \ll 1$ because the rate of change of the color charge is proportional to $g^{2}$. We therefore assume a stochastic (random phase) ansatz[17] for the expectation value of the product

$$
\left\langle[v A]_{\tau}^{b}[v A]_{\tau^{\prime}}^{d} \Lambda^{e}\left(\tau^{\prime}\right)\right\rangle \approx\left\langle[v A]_{\tau}^{b}[v A]_{\tau^{\prime}}^{d}\right\rangle\left\langle\Lambda^{e}(\tau)\right\rangle
$$

With this ansatz, eq.(9) reduces to

$$
\frac{d}{d \tau}\left\langle\Lambda^{a}(\tau)\right\rangle \approx\left\{g^{2} f^{a b c} f^{c d e} \int_{0}^{\tau} d \tau^{\prime}\left\langle[v A]_{\tau}^{b}[v A]_{\tau^{\prime}}^{d}\right\rangle\right\}\left\langle\Lambda^{e}(\tau)\right\rangle \equiv-d^{a e}(\tau)\left\langle\Lambda^{e}(\tau)\right\rangle .
$$

This defines the color diffusion tensor, $d^{a e}(\tau)$, whose physical interpretation will become clear below. Note that we cannot use the $v^{\mu} A_{\mu}^{b}=0$ gauge in Eq. (11) because $v^{\mu}(\tau)$, via eq.(6), is different for every member of the ensemble.

Our second main approximation is to work in the eikonal limit. This is equivalent to the "hard thermal loop" approximation in [9]. We thus assume that our test parton has a high initial four momentum and that energy loss via eq.(6) is small. This also follows formally from eq.(6) in the perturbative $g \ll 1$ limit. In this case, we can factor the four velocity out of the ensemble average and approximate

$$
\left\langle[v A]_{\tau}^{b}[v A]_{\tau^{\prime}}^{d}\right\rangle \approx v^{\mu} v^{\nu}\left\langle A_{\mu}^{b}(x(\tau)) A_{\nu}^{d}\left(x\left(\tau^{\prime}\right)\right)\right\rangle \equiv v^{\mu} v^{\nu} C_{\mu \nu}^{b d}\left(x(\tau), x\left(\tau^{\prime}\right)\right) .
$$


Next we note that in this eikonal limit $x^{\mu}(\tau)-x^{\mu}\left(\tau^{\prime}\right)=\left(\tau-\tau^{\prime}\right) v^{\mu}$, and thus for a homogeneous, color neutral ensemble, the correlation function $C$ can expressed as

$$
C_{\mu \nu}^{b d}\left(x(\tau), x\left(\tau^{\prime}\right)\right)=\delta_{b d} \int(d k) e^{-i(k v)\left(\tau-\tau^{\prime}\right)} C_{\mu \nu}(k, u)
$$

where $(d k)=d^{4} k /(2 \pi)^{4}, u^{\mu}$ is the four velocity of the ensemble rest frame. The correlation function $C$ measures the spontaneous fluctuations of the background field. In the classical limit those fluctuations can be calculated using kinetic theory[7, 15, 18]. For a system in thermal equilibrium, the $\omega \ll T$ spontaneous fluctuations can also be related to the response function (retarded commutator) via the fluctuation-dissipation theorem 19] as

$$
C^{\mu \nu}(k, u) \approx-\frac{2 T}{k u} \operatorname{Im} D_{R}^{\mu \nu}(k, u, T)
$$

where

$$
D_{R}^{\mu \nu}(k, u, T)=i \int d^{4} x e^{i k x} \theta\left(x_{0}\right) \operatorname{Tr}\left(\rho\left[A^{\mu}(x), A^{\nu}(0)\right]\right)
$$

is the thermal averaged retarded commutator that arises in linear response theory. The statistical operator is given by $\rho=\exp \left(-P^{\mu} u_{\mu} / T\right)$ for a thermal ensemble with four velocity $u$. Both methods give the same result in the high temperature limit in the 1-loop approximation [7]. The 1-loop result obtained in ref. [8, 9] is

$$
D_{R \mu \nu}(k, u)=-\frac{Q_{\mu \nu}}{k^{2}-\Pi_{L}}-\frac{P_{\mu \nu}}{k^{2}-\Pi_{T}}+\alpha \frac{k_{\mu} k_{\nu}}{k^{2}}
$$

where $\alpha$ is a gauge parameter. This decomposition utilizes the longitudinal and transverse projectors, $Q_{\mu \nu}=\bar{u}_{\mu} \bar{u}_{\nu} / \bar{u}^{2}$ and $P_{\mu \nu}=\bar{g}_{\mu \nu}-Q_{\mu \nu}$, as given in terms of $\bar{g}_{\mu \nu}=g_{\mu \nu}-k_{\mu} k_{\nu} / k^{2}$ and $\bar{u}_{\mu}=\bar{g}_{\mu \nu} u^{\nu}$. Furthermore, the longitudinal and transverse polarization functions are related to the gluon self energy $\Pi^{\mu \nu}(k)$ through $\Pi_{L}=\Pi^{\mu \nu} Q_{\mu \nu}$ and $\Pi_{T}=\Pi^{\mu \nu} P_{\mu \nu} / 2$.

With (14,15), the color diffusion tensor can be expressed as

$$
d^{a e}(\tau)=-2 g^{2} N T \delta_{a e} \int \frac{(d k)}{(k u)} \operatorname{Im}\left(v D_{R} v\right) \int_{0}^{\tau} d \tau^{\prime} e^{-i(k v)\left(\tau-\tau^{\prime}\right)}
$$

where the color factor for $S U(N)$ follows from $f^{a b c} f^{e b c}=N \delta_{a e}$. As $\tau \rightarrow \infty$, the proper time integral reduces to $\pi \delta(k v)$ as can be seen using $\operatorname{Im} D_{R}(-k)=-\operatorname{Im} D_{R}(k)$, to replace the limits of integration by $-\tau$ to $\tau$. We can therefore define the color diffusion coefficient, $d_{c}$, and the corresponding color relaxation time in the plasma rest frame, $t_{c}$, via

$$
\lim _{\tau \rightarrow \infty} d^{a e}(\tau) \equiv \delta_{a e} N d_{c} \equiv \delta_{a e}(v u) / t_{c}
$$

For ultra-relativistic partons, the color relaxation rate reduces to

$$
\frac{1}{t_{c}}=-2 \pi g^{2} N T \int \frac{(d k)}{(k u)} \frac{k^{2}}{(k u)^{2}-k^{2}} \delta\left(\frac{k v}{v u}\right) \operatorname{Im}\left(\frac{1}{k^{2}-\Pi_{L}}-\frac{1}{k^{2}-\Pi_{T}}\right),
$$

It is important to note that the dependence on the gauge parameter $\alpha$ dropped out because the delta function constrains $k v=0$. However, in the semi-classical limit $(\omega \ll T)$ the 
gauge invariance of $t_{c}$ holds even if $k v \neq 0$, as can be shown via kinetic theory [16, since fluctuations are driven by gauge invariant current-current correlations (polarization tensor). The gauge invariance of this result is related to the gauge invariance of the damping rate in hard thermal loop calculations in ref. [20] and depends essentially on the eikonal limit. For momenta $\sim 3 T$, the eikonal approximation applies perturbatively because the energy loss per interaction, $\sim g^{2} T$, is relatively small if $g \ll 1$.

Given $t_{c}$, the solution of eq. (11) in the plasma rest frame is

$$
\left\langle\Lambda^{a}(t)\right\rangle=e^{-t / t_{c}}\left\langle\Lambda^{a}(0)\right\rangle .
$$

Note, that while the ensemble averaged color of the parton vanishes rapidly for $t>t_{c}$, the equations of motion conserve the magnitude of the color vector, i.e., $\left\langle\Lambda^{a}(\tau) \Lambda^{a}(\tau)\right\rangle=$ constant.

We can compare the color relaxation rate, $1 / t_{c}$, to the quark or gluon damping rates noting [8] that the longitudinal and transverse polarization functions are related to the color dielectric functions via $k^{2}-\Pi_{L}=k^{2} \epsilon_{L}$ and $k^{2}-\Pi_{T}=\omega^{2} \epsilon_{T}-\vec{k}^{2}$. The analytic expressions for $\epsilon_{L}(k)$ and $\epsilon_{T}(k)$ are recorded for example in [1, 2]. In terms of these color dielectric functions

$$
\frac{1}{t_{c}}=-\frac{\alpha_{s} N T}{2 \pi^{2}} \int \frac{d^{3} k}{\omega k^{2}} \operatorname{Im}\left(\frac{1}{\epsilon_{L}(k)}+\frac{k^{2}-\omega^{2}}{\omega^{2} \epsilon_{T}(k)-k^{2}}\right)_{\omega=k \cos \theta} .
$$

This is the same integral that occurs in the quark damping rate, $\gamma_{q}$, as discussed in ref. [6]. In fact we find that it corresponds exactly to the leading log approximation to the gluon damping rate computed in [20]. This coincidence of results based on classical kinetic theory and 1-loop high T QCD is a general result [0] in all problems where the classical $\omega \ll T$ and $k \ll T$ modes of the system are dominant.

Unfortunately, as is well known[20], the transverse contribution to that damping rate is logarithmically divergent. Dynamical screening is sufficient to regulate the Coulomb divergence in the expression for the momentum relaxation rate[j] but not the damping rate. The longitudinal part is finite [6, 20] and can thus be neglected. Several attempts to deal with this problem have been proposed. One is to introduce a nonperturbative magnetic mass, $m_{M} \sim g^{2} T$, as in ref. [21]. Another is to introduce damping self consistently by adding an imaginary part to the bare fermion propagator 22]. However, no satisfactory solution is yet available 23.

In our kinetic formulation the source of the problem may be traced back to our first assumption that the field fluctuation time is short compared to the color diffusion time. What we learned a postiori was that fluctuations of the transverse magnetic fields are long ranged in time and that quasi-static unscreened magnetic fields lead to the divergent color relaxation rate. This suggests that the change of the color moment with time should taken into account within the integrand in eq.(11). Such a "memory" effect could damp the contribution from early times with $(v u)\left(\tau-\tau^{\prime}\right)>t_{c}$. Inserting a factor $\exp \left(-(v u)\left(\tau-\tau^{\prime}\right) / t_{c}\right)$ within the integral over $\tau^{\prime}$ would smear out the delta function, $\delta(k v)$, appearing in eq. (18) into a Lorentzian form $\propto d_{c} /\left((k v)^{2}+d_{c}^{2}\right)$. The resulting self consistent equation for $d_{c}$ would lead to a finite result analogous to the method considered by Altherr et al.222.

We will however follow Pisarski[20] and regulate the divergence by introducing a magnetic 
mass cutoff. In the region $\omega \ll k$, we therefore approximate

$$
\operatorname{Im}\left(\frac{1}{\omega^{2} \epsilon_{T}(k)-k^{2}}\right) \approx-\frac{a \omega / k}{\left(k^{2}+m_{M}^{2}\right)^{2}+(a \omega / k)^{2}},
$$

where $a=3 \pi \omega_{p l}^{2} / 4 \approx m_{E}^{2}$. In the $m_{M}=0$ limit this coincides with the one-loop form used for example in [6]. Note that the dynamic screening factor[5] is properly included above. With eq.(21) we can integrate the transverse part to obtain

$$
\frac{1}{t_{c}} \approx \frac{\alpha_{s} N T}{\pi} \int \frac{d k k^{2} d \mu}{\mu k^{3}} \frac{a \mu k^{2}}{\left(k^{2}+m_{M}^{2}\right)^{2}+(a \mu)^{2}} \approx \alpha_{s} N T \log \left(m_{E} / m_{M}\right)
$$

where the final form is numerically accurate already for $m_{M}<m_{E}$. The finite longitudinal part adds about 0.5 to the log which is well within the uncertainties associated with inserting a magnetic mass in (21) by hand.

To show that $d_{c}$ actually corresponds to a diffusion coefficient in kinetic theory, we consider next the classical phase space density $Q(x, p, \Lambda)$ of an ensemble of colored particles defined by

$$
Q(x, p, \Lambda)=\int d \tau^{\prime} \sum_{i} \delta\left(x-x_{i}\left(\tau^{\prime}\right)\right) \delta\left(p-p_{i}\left(\tau^{\prime}\right)\right) \delta\left(\Lambda-\Lambda_{i}\left(\tau^{\prime}\right)\right)
$$

where $p_{i}^{\mu}$ and $\Lambda_{i}^{a}$ obey Wong equations (7) coupled via a self consistent field. The phase space density obeys the (Heinz) transport equation [1, 13]:

$$
\left(p_{\mu} \partial_{x}^{\mu}+g p^{\nu} F_{\mu \nu}^{a} \Lambda^{a} \partial_{p}^{\mu}+i g p^{\mu} A_{\mu}^{a} L^{a}\right) Q(x, p, \Lambda)=0
$$

where

$$
L^{a}=-\imath f^{a b c} \Lambda^{b} \frac{\partial}{\partial \Lambda^{c}}
$$

are generators of $S U(N) / Z^{N}$ obeying the commutation relations $\left[L^{a}, L^{b}\right]=\imath f^{a b c} L^{c}$. eq Note that (24) just non-abelian Louiville equation expressing that the parton density is constant along a characteristic, $(x(\tau), p(\tau), \Lambda(\tau))$, satisfying the Wong equation in the self consistent field, i.e.,

$$
\frac{d}{d \tau} Q(x(\tau), p(\tau), \Lambda(\tau))=-\int d \tau^{\prime} \frac{d}{d \tau^{\prime}} \sum_{i} \delta\left(x(\tau)-x_{i}\left(\tau^{\prime}\right)\right) \cdots=0
$$

The ensemble average of eq. (26) can be expressed as

$$
m \frac{d}{d \tau}\langle Q(x(\tau), p(\tau), \Lambda(\tau))\rangle=-\imath g\left\langle p^{\mu} \delta A_{\mu}^{a} L^{a} \delta Q\right\rangle-g\left\langle p^{\nu} \delta F_{\mu \nu}^{a} \Lambda^{a} \partial_{p}^{\mu} \delta Q\right\rangle,
$$

where we decompose $Q=\langle Q\rangle+\delta Q$ and $A=\langle A\rangle+\delta A$, and the characteristic trajectory satisfies

$$
m \frac{d x^{\mu}}{d \tau}=p^{\mu} \quad, \quad \frac{d p^{\mu}}{d \tau}=g v_{\nu}\left\langle F_{a}^{\mu \nu}\right\rangle \Lambda^{a} \quad, \frac{d \Lambda^{a}}{d \tau}=g f^{a b c} v^{\mu}\left\langle A_{\mu}^{b}\right\rangle \Lambda^{c}
$$


To proceed, we assume average color neutrality, $\langle A\rangle=\langle F\rangle=0$, and neglect the momentum degradation part of the kinetic equation to focus exclusively on color diffusion. Therefore, the mean phase space density evolves according to

$$
m \frac{d}{d \tau}\langle Q(x(\tau), p, \Lambda)\rangle=-\imath g\left\langle v^{\mu} \delta A_{\mu}^{a} L^{a} \delta Q\right\rangle .
$$

The kinetic equation for the fluctuating part of the phase space density is obtained by subtracting eq.(27) from .(26):

$$
\frac{d}{d \tau} \delta Q(x(\tau), p(\tau), \Lambda(\tau))=-\imath g[v \delta A]_{\tau}^{a} L^{a}\langle Q\rangle-g v^{\nu} \delta F_{\mu \nu}^{a} \Lambda^{a} \partial_{p}^{\mu}\langle Q\rangle,
$$

where in this case

$$
\frac{d p^{\mu}}{d \tau}=g v_{\nu}\left(\left\langle F_{a}^{\mu \nu}\right\rangle+\delta F_{a}^{\mu \nu}\right) \Lambda^{a}, \frac{d \Lambda^{a}}{d \tau}=g f^{a b c} p^{\mu}\left(\left\langle A_{\mu}^{b}\right\rangle+\delta A_{\mu}^{b}\right) \Lambda^{c} .
$$

Taking into account our assumption $\langle A\rangle=\langle F\rangle=0$, neglecting the induced fluctuations caused by the gradient in momentum, and retaining only terms linear in fluctuating quantities Eq. (30) reduces to

$$
\frac{d}{d \tau} \delta Q(x(\tau), p, \Lambda)=-\imath g[v \delta A]_{\tau}^{a} L^{a}\langle Q\rangle,
$$

This linearized approximation implicitely assumes the eikonal limit since then $p$ is constant and also $\Lambda$ is constant to that order. Thus both the assumptions made in deriving the color diffusion coefficient previously correspond to this linearization of the kinetic equations for fluctuations. Integrating eq.(32) and substituting the induced fluctuation into eq.(29) leads to

$$
\frac{d\langle Q\rangle}{d \tau}=-g^{2} \int_{-\infty}^{\tau} d \tau^{\prime}\left\langle[v \delta A]_{\tau}^{a}[v \delta A]_{\tau^{\prime}}^{b}\right\rangle L^{a} L^{b}\left\langle Q\left(x\left(\tau^{\prime}\right), p, \Lambda\right)\right\rangle .
$$

Assuming that fluctuation correlation time is short compared to the color diffusion time, $\left\langle Q\left(x\left(\tau^{\prime}\right), p, \Lambda\right)\right\rangle \approx\langle Q(x(\tau), p, \Lambda)\rangle$ allowing us to factor $Q$ out of the integrand. In this way, we obtain for homogeneous and color neutral ensemble the following color diffusion equation

$$
\frac{d}{d \tau}\langle Q(x(\tau), p, \Lambda)\rangle=-d_{c} L^{a} L^{a}\langle Q\rangle,
$$

where color diffusion coefficient $d_{c}$ is given by

$$
d_{c}=g^{2} v^{\mu} v^{\nu} \int_{-\infty}^{0} d \tau^{\prime} C_{\mu \nu}\left(x(0), x\left(\tau^{\prime}\right)\right),
$$

where $C$ is given by eqs.(12-15), is thus the same color diffusion coefficient that we derived via eqs.(16- 22)). Note that Eq. (34) can be written in the form of Fokker-Planck equation

$$
\frac{d\langle Q\rangle}{d \tau}=-\frac{\partial}{\partial \Lambda^{a}}\left(d^{a b} \frac{\partial}{\partial \Lambda^{b}}\langle Q\rangle\right),
$$


where color diffusion coefficient $d^{a b}$ is given by

$$
d^{a b}=d_{c} f^{a d c} f^{c e b} \Lambda^{d} \Lambda^{e}
$$

Equation (34) therefore describes diffusion in color space. Note that operator $C_{2}=L^{a} L^{a}$ just is quadratic Casimir operator of $S U(N) / Z^{N}$. For a more detailed derivation of collision terms including momentum space diffusion we refer to [14, 15, 16].

To illustrate color diffusion, it is instructive to consider $S U(2)$, where $L^{i}=-\imath \epsilon^{i j k} \Lambda^{j} \partial / \partial \Lambda^{k}$ is an angular momentum operator in color (iso-spin) space and $C_{2}=l(l+1)$. Since the magnitude of $\vec{\Lambda}$ is fixed, any initial distribution in color space can be expanded in the spherical harmonics as

$$
\left\langle Q(\vec{\Lambda}, \tau=0)=\sum_{l, m} c_{l, m} Y_{l}^{m}(\theta, \phi)\right.
$$

where $\theta, \phi$ are polar coordinates in iso-spin space. In this case, the proper time evolution is simply

$$
\langle Q(\vec{\Lambda}, \tau)\rangle=\sum_{l, m} c_{l, m} Y_{l}^{m}(\theta, \phi) e^{-d_{c} \tau l(l+1)}
$$

From Eq. (39) it follows that any non-isotropic distribution in color space evolves to a uniform distribution,

$$
\lim _{\tau \rightarrow \infty}\langle Q(\vec{\Lambda}, \tau)\rangle=c_{0} / \sqrt{ } 4 \pi
$$

on a proper time scale $\sim 1 / 2 d_{c}$ (for $N=2$ ). The same picture holds qualitatively for the general $S U(N)$ case, where any distribution evolves to uniform distribution which is determined by the zero mode of quadratic Casimir operator. This color equilibrium state is achieved after a proper $\tau_{c}=1 / N d_{c}$. Since the time in the plasma rest frame is $t=(v u) \tau$ and $N d_{c}=(v u) / t_{c}$, color equilibrium in the plasma rest frame is achieved on the time scale $t_{c}$.

Finally, to calculate the color conductivity coefficient, we must test the linear response of the system to a weak external field. In an external filed, $F_{e x}$, the kinetic equation for the mean density must be supplemented by a Vlasov term, $g p F_{e x} \Lambda \partial_{p}\langle Q\rangle$, on the left hand side of Eqs.(29,34). To calculate the induced color current, we multiply that modified Eq.(34) with $\Lambda^{a}$ and integrate over the color charge with the measure [13]

$$
d \Lambda \propto \prod_{i=1}^{N^{2}-1} d \Lambda_{i} \delta\left(\Lambda^{a} \Lambda_{a}-q_{2}\right) \delta\left(\Lambda^{a} \Lambda^{b} \Lambda^{c} d_{a b c}-q_{3}\right) .
$$

The proportionality constant is chosen such that [13] $\int d \Lambda=1, \int d \Lambda \Lambda^{a}=0$, and $\int d \Lambda \Lambda^{a} \Lambda^{b}=$ $c_{2} \delta^{a b}$. The charges are defined through the trace of the Casimir operators: $q_{2}=\operatorname{Tr}\left(t^{a} t^{a}\right)$, $q_{3}=d_{a b c} \operatorname{Tr}\left(t^{a} t^{b} t^{c}\right)$. For quarks $q_{2}=\left(N^{2}-1\right) / 2, q_{3}=\left(N^{2}-4\right)\left(N^{2}-1\right) / 4 N$ and $c_{2}=1 / 2$. For gluons $q_{2}=N\left(N^{2}-1\right), q_{3}=0, c_{2}=N$.

This leads to the following linearized kinetic equation for the adjoint color moment density, $q^{a}(x, p)$,

$$
v^{\mu} \partial_{\mu} q^{a}+c_{2} g v^{\mu}\left[F_{e x}\right]_{\nu \mu}^{a} \partial_{p}^{\nu}\langle Q\rangle_{0}=-N d_{c} q^{a}
$$


where

$$
q^{a}(p, x)=\int d \Lambda \Lambda^{a}\langle Q(x, p, \Lambda)\rangle
$$

For a homogeneous, color neutral ensemble, $\langle Q(p, x, \Lambda)\rangle=\langle Q(p)\rangle_{0}$, and thus since $\int d \Lambda \Lambda^{a}=$ 0 the color moment density vanishes. However, in a weak external field there is a distortion of the color density linear in $F_{\text {ex }}$. The factor $N$ on the right hand side of (42) follows from $\int d \Lambda \Lambda^{a} L^{2} Q=N q^{a}$, which can be verified using $\left[L^{a}, \Lambda^{b}\right]=\imath f^{a b c} \Lambda^{c}$ and the property that $\int d \Lambda L^{a} F(\Lambda)=0$, which follows upon integration by parts and noting the symmetry properties of $f^{a b c}$ and $d^{a b c}$. We note also that in contrast to (42) the kinetic equation for the color singlet density, $q(x, p)=\int d \Lambda\langle Q\rangle$, is independent of the color diffusion coefficient.

Solving (42) for the Fourier transform, $q^{a}(k, p)$, the induced color current is given by

$$
j^{\mu a}(k)=g \int d p p^{\mu} q^{a}(p, k) \equiv \sigma^{\mu \alpha \beta}(k)\left[F_{e x}(k)\right]_{\alpha \beta}^{a},
$$

where the conductivity tensor for a homogeneous color neutral plasma of quarks, antiquarks, and gluons is [7, 2]

$$
\sigma^{\mu \alpha \beta}(k)=g^{2} \int d p \frac{p^{\mu} p^{\alpha}}{-i(p k)+(p u) / t_{c}} \partial_{p}^{\beta}\left\{\frac{N_{f}}{2}\left(f_{q}(p)+f_{\bar{q}}(p)\right)+N f_{g}(p)\right\},
$$

where, $f_{i}(p)$ are the momentum space distributions including spin degeneracy for the partons. For example, in equilibrium $f_{g}(p)=\left(2 /(2 \pi)^{3}\right) 2 \theta\left(p_{0}\right) \delta\left(p^{2}\right) /\left(e^{p u / T}-1\right)$. For an isotropic plasma this tensor reduces to $\sigma^{\mu \alpha \beta}=\sigma^{\mu \nu}(k) u^{\beta}$. Finally, from Eq. (45) it follows that [7, 2] the static $(k=0)$ color conductivity is $\sigma^{\mu \nu}(0)=\sigma_{c} g^{\mu \nu}$, where

$$
\sigma_{c}=t_{c} \omega_{p l}^{2}
$$

The important difference between our derivation and that in ref. [7, 2] is that the collision rate is derived here directly from the kinetic equation for color fluctuations rather than parameterized via the relaxation time approximation. We see in particular that the color diffusion time rather than momentum degradation time controls the magnitude of the conductivity. In addition, the same color diffusion rate applies for both quarks and gluons unlike in the general ansatz of [2]. Inserting the expression for $t_{c}$, we thus find the surprising result stated in the introduction, i.e., that

$$
\sigma_{c} \approx 2 T / \log \left(m_{E} / m_{M}\right)
$$

The appearance of the non-perturbative and non-classical magnetic mass in this final expression suggests that the classical $(k \rightarrow 0)$ or high $T$ limit of a QGP is in fact be very different from QED plasmas because of color diffusion. Another indication of this is that the long wavelength $(k \ll T)$ color plasmon mode, obtained from the dispersion relation $k^{2}=\Pi_{L}(k)=-i Q_{\mu \nu}\left(\sigma^{\mu \alpha \nu}-\sigma^{\mu \nu \alpha}\right) k_{\alpha}$, are strongly damped with a rate proportional to $1 / t_{c}$ as can be seen from the general discussion in ref. [7, 2]. Thus, the anomalously large damping rate of hard thermal partons propagates down to the soft collective modes. Probably not only the plasmon but also the plasmino are over-damped due to color diffusion. In this respect, further study of color diffusion and screening of transverse interactions are very important in the development of QGP transport theory. In practical applications, especially to nuclear 
collisions, of course all time integrals are restricted to a finite time, which automatically regulates the formal static divergences. The problem in that connection is to determine which

effect, finite time or nonperturbative magnetic screening, restricts the magnitude of color diffusion the most.

Acknowledgments: We thank R. Pisarski, S. Gavin, and T.Ludlam for fruitful discussions.

\section{References}

[1] H.-Th. Elze and U. Heinz, Phys. Rep. 183 (1989) 81; U. Heinz, Phys. Rev. Lett., 51 (1983) 351; H.-Th. Elze, M. Gyulassy and D. Vasak, Nucl. Phys. B276 (1986) 706; Phys. Lett. 177B (1986) 402.

[2] S. Mrówczynski, in Quark-Gluon Plasma, ed. R. Hwa, World Scientific, 1990; Phys.Rev. D39 (1989) 1940.

[3] See refs. in Proc. of Quark Matter 91, Nucl. Phys. A544 (92) 1c.

[4] P. Danielewicz and M. Gyulassy, Phys. Rev. D31 (1985) 53; A. Hosoya and K. Kajantie, Nucl. Phys. B250 (1985) 666.

[5] G. Baym, et al. Phys. Rev. Lett. 64 (1990) 1867.

[6] M. Thoma, M. Gyulassy, Nucl. Phys. B351 (1991) 491; E. Braaten, M. Thoma, PRD 44 (1991) 1298; R2625; M. Gyulassy, et al. Nucl. Phys. A 538 (1992) 37c.

[7] U. Heinz, Ann. Phys. (N.Y.) 168 (1986) 148;

[8] H.A. Weldon, Phys. Rev., D26 (1982) 1394; V.V. Klimov, Sov. J. Nucl. Phys. 33 (1981) 934; E.V. Shuryak, JETP 47 (1978) 212.

[9] E. Braaten, R. Pisarski, Phys.Rev.Lett. 64 (1990) 1338; Nucl.Phys. B337 (1990) 569.

[10] G. Gatoff, A. K. Kerman and T. Matsui, Phys. Rev. D36 (1987) 114.

[11] K.J. Eskola and M. Gyulassy, Phys.Rev. C47 (1993) 2329.

[12] S.K. Wong, Nuovo Cim. 65A (1970) 689.

[13] U. Heinz, Ann. Phys. (N.Y.) 161 (1985) 48.

[14] A.V. Selikhov, Phys. Lett., B268 (1991) 263; Erratum B285 (1992) 398.

[15] A.V. Selikhov, Preprint IAE-5526/1, Moscow,1992; to be submitted to Nucl. Phys. B.

[16] A.V. Selikhov and M. Gyulassy, in preparation.

[17] N.G. van Kampen, Phys. Rep. C24 (1976) 171. 
[18] V.P. Silin, Sov.Phys. J.E.T.P. 11 (1960) 1136.

[19] H.B. Callen and T. A. Welton, Phys.Rev. 83 (1951) 34; W.B. Thompson and J. Hubbard, Rev.Mod.Phys. 32 (1960) 714; J. Hubbard Proc. Roy. Soc. A260 (1961) 114.

[20] R.D. Pisarski, Phys.Rev.Lett. 63 (1989) 1129; R.D. Pisarski, BNL-P-1/92, unpublished.

[21] A. Linde, Phys. Lett. 93B (1980) 327; D. J. Gross, R.D. Pisarski, L.G.Yaffe, Rev.Mod.Phys. 53 (1981) 43.

[22] V.V. Lebedev and A.V. Smilga, Ann.Phys. (N.Y.) 202 (1990) 229; T. Altherr, E. Petitgirard, T. del Rio Gaztellurutia, Phys. Rev. D47 (1993) 703; R. Kobes, G. Kunstatter, K. Mak, PRD45 (1990) 4632;

[23] S. Peigne, E. Pilon, D. Schiff, LPTHE-Orsay-93/13 unpublished. 